\title{
Ontogenetic growth: models and theory
}

\author{
Anastassia M. Makarieva ${ }^{a}$, Victor G. Gorshkov ${ }^{a}$, Bai-Lian Li ${ }^{b, *}$ \\ a Theoretical Physics Division, Petersburg Nuclear Physics Institute, Russian Academy of Sciences, 188300 Gatchina, St.-Petersburg, Russia \\ ${ }^{\mathrm{b}}$ Department of Botany and Plant Sciences, Ecological Complexity and Modeling Laboratory, \\ University of California, Riverside, CA 92521-0124, USA
}

Received 6 January 2003; received in revised form 22 August 2003; accepted 16 September 2003

\begin{abstract}
We re-analyze the assumptions underlying two recently proposed ontogenetic growth models [Nature 413 (2001) 628; Nature 417 (2002) 70] to find that the basic relations in which these models are grounded contradict the law of energy conservation. We demonstrate the failure of these models to predict and explain several important lines of empirical evidence, including (a) the organismal energy budget during embryonic development; (b) the human growth curve; (c) patterns of metabolic rate change during transition from embryonic to post-embryonic stages; and (d) differences between parameters of embryonic growth in different taxa. We show how a theoretical approach based on well-established ecological regularities explains the observations where the formal models fail. Within a broader context, we also discuss major principles of ontogenetic growth modeling studies in ecology, emphasizing the necessity of ecological theory to be based on assumptions that are testable and to be formulated in terms of variables and parameters that are measurable.
\end{abstract}

(C) 2003 Published by Elsevier B.V.

Keywords: Ontogenetic growth; Modeling; Energy conservation; Metabolic rate; Embryo; Growth efficiency

\section{Introduction}

Recently, the interest in ontogenetic growth modeling has conspicuously revived. In two recent papers, a general framework was proposed for description of ontogenetic development of different organisms at different temperatures (West et al., 2001; Gillooly et al., 2002). This framework claimed to be derived from basic physical and biological principles. The models are based on what the authors refer to as the conservation of energy equation:

$B=N B_{\mathrm{c}}+\frac{E_{\mathrm{c}} \mathrm{d} N}{\mathrm{~d} t}$

\footnotetext{
* Corresponding author. Tel.: +1-909-787-4776, fax: +1-909-787-4437.

E-mail address: bai-lian.li@ucr.edu (B.-L. Li).
}

Here $B$ is the average resting metabolic rate of the whole organism, interpreted as the incoming rate of energy flow (West et al., 2001); $N$ is the total number of cells in the organism, $B_{\mathrm{c}}$ is the metabolic rate of a single cell, $t$ is time, $E_{\mathrm{c}}$ is defined as metabolic energy needed to create a cell, $B_{\mathrm{c}}$ and $E_{\mathrm{c}}$ are assumed to be fundamental constants which are independent of $N$. Using mammals as an example, West et al. (2001) calculate $E_{\mathrm{c}}$ by multiplying the average energy content of mammalian tissue $\left(E=7 \times 10^{3} \mathrm{Jg}^{-1}\right)$ by average cell mass $\left(m_{\mathrm{c}}=3 \times 10^{-9} \mathrm{~g}\right)$, thus arriving at the cell energy content, $E_{\mathrm{c}}=2.1 \times 10^{-5} \mathrm{~J}$.

According to Eq. (1), the rate at which the energy content of the organism is growing, $B_{\mathrm{g}} \equiv E_{\mathrm{c}} \mathrm{d} N / \mathrm{d} t$, is always less than the total metabolic energy of the organism $B$, due to maintenance expenses $B_{\mathrm{c}} N>0$, i.e., $B_{\mathrm{g}} / B<1$. Experimental data show that this is 
Table 1

Relative energy expenses during development from fertilization to hatching for various organisms ${ }^{\mathrm{a}}$

\begin{tabular}{|c|c|c|c|}
\hline Organism & $B_{\mathrm{g}} / B$ & $\varepsilon$ & Source \\
\hline \multicolumn{4}{|l|}{ Birds } \\
\hline Chicken & 1.65 & 0.61 & Brody (1945) \\
\hline \multicolumn{4}{|l|}{ Amphibia } \\
\hline Frog & $1.0-3.0$ & $0.51-0.75$ & Brody (1945) \\
\hline \multicolumn{4}{|l|}{ Fish } \\
\hline Solea senegalensis & 6.1 & 0.86 & Yúfera et al. (1999) \\
\hline Solea solea & $0.9-2.1$ & $0.47-0.68$ & Yúfera et al. (1999) \\
\hline Clupea harengus & 2.3 & 0.70 & Yúfera et al. (1999) \\
\hline Sparus aurata & 10.1 & 0.91 & Yúfera et al. (1999) \\
\hline Clarias gariepinus & 9.0 & 0.90 & Yúfera et al. (1999) \\
\hline \multicolumn{4}{|l|}{ Echinoderms } \\
\hline Fundulus heteroclitus & 1.4 & 0.59 & Brody (1945) \\
\hline \multicolumn{4}{|l|}{ Crustaceans } \\
\hline Cyclograpsus lavauxi & $\sim 1.7$ & $\sim 0.6$ & Taylor and Leelapiyanart (2001) \\
\hline Heterozius rotundifrons & $\sim 2.4$ & $\sim 0.7$ & Taylor and Leelapiyanart (2001) \\
\hline \multicolumn{4}{|l|}{ Insects } \\
\hline Silk-worm & 1.7 & 0.63 & Brody (1945) \\
\hline
\end{tabular}

${ }^{a}$ Notations: $B_{\mathrm{g}}$, energy content of tissues of a pre-hatching embryo; $B$, cumulative metabolic expenses of the embryo from fertilization to hatching; $\varepsilon$, growth efficiency.

not true for embryonic development of various organisms (Table 1). For example, during development from egg fertilization to hatching a chicken embryo expends $B=97 \mathrm{~kJ}$, while it accumulates $B_{\mathrm{g}}=160 \mathrm{~kJ}$ in its body. This gives $B_{\mathrm{g}} / B=160 / 97=1.65$. Corresponding values in silk-worm, sea urchin and crabs range from 1.4 to 2.4 , rising up to 10 in some fishes (Table 1).

The violation of the energy conservation law illustrates that relation (Eq. (1)) is inappropriate. This calls for revision of the ontogenetic growth models and their implications that ground in Eq. (1). We perform such a critical analysis on the basis of the available empirical data. Using well-established ecological relations and directly measurable variables we account for major regularities governing the ontogenetic growth process and discuss general principles of theoretical studies in this field.

\section{Basic equations of ontogenetic models}

The energy spent by the organism to construct a cell cannot be estimated by the cell energy content, as done by West et al. (2001). Animal tissues are not synthesized anew from inorganic substances, but from energy-rich organic blocks (e.g., amino-acids) arriving into the organism with food. Food may be either gathered in the environment or prepared by the maternal organism (e.g., milk, food stores of the egg, etc.). Depending on the characteristics of material influx and properties of tissues being built, the energy needed to convert the organic matter of food into living tissues may be arbitrarily high or low. Except for energy content per cell there are no other fundamental cell constants with appropriate dimension, while this constant, as we have shown in Section 1, cannot be embodied into the model of West et al. (2001) without violating the energy conservation law. We therefore conclude that the claim made by West et al. (2001) that all parameters of their model can be "independently determined from fundamental parameters of the cell" remains unsupported. Furthermore, there are no physical or biological grounds to assume that energy needed to create one cell of the organism is independent of body mass. A larger organism could spend more to make one cell than a smaller one, or vice versa.

Another interpretation of Eq. (1) can be found in the works of von Bertalanffy (see, for example, von Bertalanffy (1957)). Multiplying all parts of Eq. (1) by 
$m_{\mathrm{c}} / E_{\mathrm{c}}$, where $m_{\mathrm{c}} \equiv m / N$ is average cell mass, and representing resting metabolic rate $B$ in the well-known allometric form, $B=B_{0} m^{\alpha}$, we arrive at the so-called von Bertalanffy equation:

$\frac{\mathrm{d} m}{\mathrm{~d} t}=a m^{\alpha}-b m$,

where $a=B_{0}\left(m_{\mathrm{c}} / E_{\mathrm{c}}\right), b=B_{\mathrm{c}} / E_{\mathrm{c}}$ are $m$-independent constants, if one assumes that $B_{0}, B_{\mathrm{c}}$ and $E_{\mathrm{c}}$ are also $m$-independent. The first and second terms in the right-hand side of Eq. (2) were interpreted by von Bertalanffy as the rates of synthesis and decomposition of organic matter, respectively. Eq. (2) thus took the meaning of the law of matter conservation for organic matter. For a particular value of $\alpha=2 / 3$, the first term in the right-hand side of Eq. (2) can be interpreted as the rate of food intake under the assumption that it is proportional to body surface area $S \propto \mathrm{m}^{2 / 3}$ (see, e.g., Kooijman, 2000; Nisbet et al., 2000). This assumption is plausible on a larger ecological scale (e.g., in interspecific comparisons of evolutionarily related organisms), but it is not self-evident when applied to ontogenetic growth. A smaller but intensively growing organism may well uptake a different amount of energy per unit surface area than a larger but non-growing one. von Bertalanffy did not insist on any particular physical or biological argumentation for the proposed dependence (Eq. (2)) of synthesis and decomposition of organic matter on body mass $m$; he only considered $\mathrm{a}$ and $\mathrm{b}$ as fitting parameters of his model and justified the model mainly by the goodness-of-fit. West et al. (2001) admitted the formal similarity between their model equation and that of von Bertalanffy with $\alpha=3 / 4$, but stated that the difference lies in that the parameters of their model "are directly calculable from fundamental cellular parameters". This claim remaining unsupported, as we have shown, the model of West et al. (2001) appears identical to that of von Bertalanffy (1957).

The inadequacy of the assumption of constant $B_{\mathrm{c}}$ for the whole life-span of the organism (West et al., 2001; Gillooly et al., 2002) and, consequently, constant coefficient $b$ in von Bertalanffy Eq. (2), becomes explicit when considering endothermic animals. As we have seen, the term $E_{\mathrm{c}} \mathrm{d} N / \mathrm{d} t$ in Eq. (1) is assumed to be equal to the increment of organismal energy content. That is, this term describes the flux of ordered energy, which does not dissipate but is deposited within the organism's tissues. Therefore, the heat flux produced $H_{\mathrm{p}}$ and dissipated $H_{\mathrm{d}}$ by the heat-balanced organism are both equal to $B_{\mathrm{c}} N$. The necessity to maintain constant body temperature demands that $H_{\mathrm{d}}$ be proportional to the organism's body surface area $S$, which in endothermic animals scales with body mass $m$ as $S \propto$ $m^{\alpha}, \alpha \sim 0.6-0.8$ (Economos, 1982), so that $H_{\mathrm{d}} \propto m^{\alpha}$. On the other hand, it follows from the assumed constancy of $B_{\mathrm{c}}$ in Eq. (1) that $H_{\mathrm{d}} \equiv B_{\mathrm{c}} N$ is directly proportional to body mass $m, H_{\mathrm{d}} \propto m$, as far as $N \propto m$. This contradiction essentially means that $\left(H_{\mathrm{p}} / H_{\mathrm{d}}\right)_{\alpha<1}>1$ if $m<m_{1}$ and $\left(H_{\mathrm{p}} / H_{\mathrm{d}}\right)_{\alpha<1}<1$ if $m>m_{1}$, where mass $m_{1}$ is equal to body mass of a heat-balanced organism, for which $H_{\mathrm{d}}=H_{\mathrm{p}}$. It follows that if one fixes the value of $B_{\mathrm{c}}$ for a heat-balanced endothermic organism with body mass $m_{1}$, one comes to the conclusion that the younger (smaller) organisms with $m<m_{1}$ and older (larger) organisms with $m>m_{1}$ should be unable to sustain their characteristic body temperature, suffering from overcooling and overheating, respectively.

\section{Energy conservation equation for ontogenetic growth}

As is well-known in ecology (Brody, 1945; Odum, 1971; Whittaker, 1975; Pandian and Vernberg, 1987; Kooijman, 2000), the incoming energy flux for the organism is not its metabolic rate $B$, as suggested by West et al. (2001), but the energy flux of the assimilated food $F$ (energy content of ingested food minus energy content of excreta, per unit time). This energy is partitioned between respiration, i.e., decomposition of organic matter which corresponds to metabolic rate $B$, and organic synthesis, which corresponds to growth of the organism at the rate of $\mathrm{d} E / \mathrm{d} t$, where $E$ is the cumulative energy content of the organism. The conservation of energy equation for animals thus reads:

$F=B+\frac{\mathrm{d} E}{\mathrm{~d} t}$

All the terms in Eq. (3) are directly measurable. Energy content of organic matter (food, living biomass and excreta) is conventionally measured as heat energy released in the course of combustion. Respiration $B$ (W per individual) in animals is measured on the basis of the rate of $\mathrm{O}_{2}$ uptake or $\mathrm{CO}_{2}$ release. Major 
constituents of animal bodies are proteins and lipids. These organic compounds have quite different combustion values, $E_{\mathrm{p}}=5.7$ and $E_{1}=9.4 \mathrm{kcal} \mathrm{g}^{-1}$, respectively (Pike and Brown, 1975). However, it takes more $\mathrm{O}_{2}$ molecules to combust $1 \mathrm{~g}$ of lipids than $1 \mathrm{~g}$ of proteins. As a result, heat release per one molecule of $\mathrm{O}_{2}$ during combustion of proteins and lipids appears to approximately coincide, $4.6 \mathrm{kcall}^{-1} \mathrm{O}_{2}$ (or $\mathrm{CO}_{2}$ ) (Brody, 1945; Pike and Brown, 1975). Therefore, irrespective of body composition of the animal (which may be subject to considerable changes, see, e.g., Worthy and Lavigne, 1983 and Kooijman, 2000), the rate of respired oxygen unambiguously determines the rate at which the energy content of the organism is changing due to metabolic processes. We also note that the heat release per one $\mathrm{O}_{2}$ molecule for carbohydrates is not essentially different from that for lipids and proteins, $5.1 \mathrm{kcall}^{-1} \mathrm{O}_{2}$. The energy combustion value of carbohydrates is about $4 \mathrm{kcal} \mathrm{g}^{-1}$.

To characterize energy expenses allocated to growth, one introduces growth efficiency $\varepsilon$ (Brody, 1945; Pandian and Vernberg, 1987; Arnould et al., 1996; del Giorgio and Cole, 1998; Parra and Yúfera, 2001), which, among other possible ways, can be defined as the ratio between the assimilated food flux and energy increment of the growing organism, $\mathrm{d} E / \mathrm{d} t \equiv \varepsilon F, 0 \leq \varepsilon<1$. From (3) we have

$\frac{\mathrm{d} E}{\mathrm{~d} t}=\frac{\varepsilon}{1-\varepsilon} B$

When $\varepsilon \sim 1$, the organism may grow rapidly at very low metabolism, $\mathrm{d} E / \mathrm{d} t \gg B$. A major determinant of $\varepsilon$ is the diet (Brody, 1945). The food stores of the egg are purposely fitted for embryos' needs and can be converted into embryonic tissues at exceptionally low expense, $\varepsilon=\varepsilon_{\max } \sim 0.7$ (Table 1). This explains why the rate of increase of organic matter content in embryos significantly exceeds the value of $B$ (Table 1 ), as discussed above.

One can write Eq. (4) in dimensionless variables:

$$
\begin{aligned}
& \frac{1}{\mu} \frac{\mathrm{d} \mu}{\mathrm{d} \tau}=\frac{\varepsilon(\mu)}{1-\varepsilon(\mu)}, \quad \mu \equiv \frac{m}{M}, \\
& \mathrm{~d} \tau \equiv \mathrm{d} t \theta, \quad \theta \equiv \frac{B}{E_{\mathrm{m}} m},
\end{aligned}
$$

where $M$, as above, is the species-specific value of maximum mass attained by individuals, $E_{\mathrm{m}} \equiv E / m$ is the mass-specific energy content of living body, $\tau \equiv$ $\int_{0}^{t}\left(B\left(t^{\prime}\right) /\left(E_{\mathrm{m}}\left(t^{\prime}\right) m\left(t^{\prime}\right)\right)\right) \mathrm{d} t^{\prime}$ is physiological age of the organism (Brody, 1945). The mean value of $\theta^{-1}$ gives the mean residence time of chemical elements in the living body. The instantaneous value of $\theta^{-1}$ is proportional to the minimum time interval "discerned" by the organism at a given stage of its development.

In Eq. (5) we assumed that the mass-specific energy content of living biomass $E_{\mathrm{m}} \equiv E / m$ is approximately constant, to facilitate comparisons with models of West et al. (2001) and Gillooly et al. (2002), where the assumption of constant $E_{\mathrm{m}}$ is also adopted, by assuming constancy of $E_{\mathrm{c}}$ and $m_{\mathrm{c}}$, see Eq. (1), $E_{\mathrm{m}} \equiv E_{\mathrm{c}} / m_{\mathrm{c}}$. That is, to go from Eq. (4) containing $\mathrm{d} E / \mathrm{d} t$ to Eq. (5) containing $\mathrm{d} m / \mathrm{d} t$ we put $\mathrm{d} E / \mathrm{d} t \equiv$ $m \mathrm{~d} E_{\mathrm{m}} / \mathrm{d} t+E_{\mathrm{m}} \mathrm{d} m / \mathrm{d} t \approx E_{\mathrm{m}} \mathrm{d} m / \mathrm{d} t$.

In order to obtain a more precise quantitative picture of general patterns in ontogenetic growth, the dynamics of $E_{\mathrm{m}}$ change in different organisms should be investigated more closely and the $m \mathrm{~d} E_{\mathrm{m}} / \mathrm{d} t$ term retained while obtaining the derivative of $E$ over time. A detailed account of $E_{\mathrm{m}}$ change in ontogeny can be found in the works of Kooijman (2000). However, for our purposes of analyzing the top major factors controlling the ontogeny of organismal energetics, Eq. (5) is sufficient.

In terms of $\tau$ and $\mu$, growth curves $\mu(\tau)$ are completely determined by $\varepsilon(\mu)$ and will coincide for all animals sharing a similar dependence $\varepsilon(\mu)$, both ectothermic and endothermic, irrespective of how their metabolic rate depends on mass (Brody, 1945). During post-embryonic growth under natural conditions, growth efficiency $\varepsilon$ is reported to decrease towards maturity from values of $0.2-0.4$ to less than $0.10-0.05$ in taxa as different as mammals, birds, bivalvia, gastropods, crustaceans and flatworms (Brody, 1945; Pandian and Vernberg, 1987). In Fig. 1, a typical $\varepsilon(\mu)$ curve is shown based on the data for hen and cow.

The growth curve $\mu(\tau)$ can be obtained by integrating Eq. (5) given that $\varepsilon(\mu)$ is known:

$$
\begin{aligned}
& \tau=\int_{0}^{\mu} \frac{\mathrm{d} \mu^{\prime}}{\mu^{\prime} \varphi\left(\mu^{\prime}\right)} \equiv \Phi(\mu), \\
& \varphi(\mu) \equiv \frac{\varepsilon(\mu)}{1-\varepsilon(\mu)}, \quad \mu=\Phi^{-1}(\tau),
\end{aligned}
$$

where $\Phi^{-1}(\tau)$ is the reverse function of $\Phi(\mu)$. During post-embryonic development at $\mu>\mu_{0}$ ( $\mu_{0}$ is 


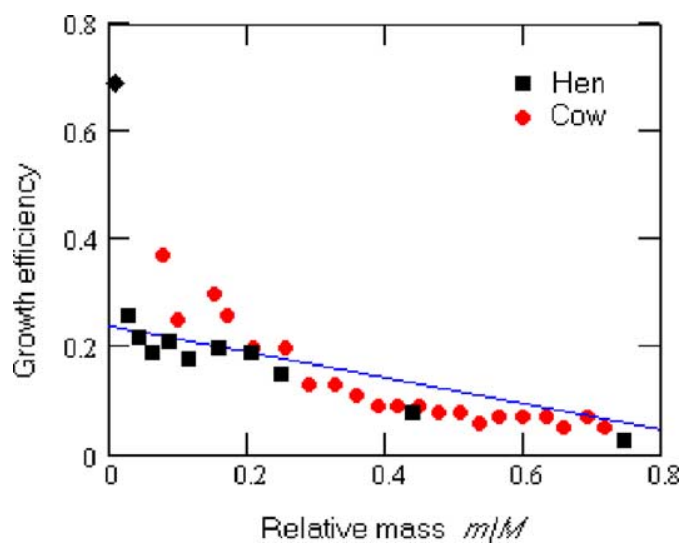

Fig. 1. Post-embryonic growth efficiency $\varepsilon$ for hen and cow versus relative mass $\mu \equiv m / M$. Data are compiled from Brody (1945). For cow $M=500 \mathrm{~kg}$, for hen $M=2 \mathrm{~kg}$. The diamond indicates growth efficiency of a pre-hatching chicken embryo. Elevated values of $\varepsilon$ for cow at low $\mu$ presumably correspond to the period of lactation, i.e., consumption of food specially designed for it. The straight line represents linear approximation $\varepsilon=\varepsilon_{0}(1-\mu), \varepsilon_{0}=0.24 \pm 0.01$.

the relative mass at birth), the growth efficiency $\varepsilon(\mu)$ is significantly less than unity, $\varepsilon(\mu) \ll 1$. In such a case function $\varphi(\mu) \approx \varepsilon(\mu)$ can be reasonably well approximated by a linear function, which takes into account cessation of growth at the moment of attaining the maximum mass, $\mu=1$. (A linear regression of $\varepsilon$ on $\mu$ with a single parameter $\varepsilon_{0}$ of the pooled data for hen and cow in Fig. 1, yielded $\varepsilon_{0}=0.24 \pm 0.01, r^{2}=$ 0.70 with 30 d.f. We note, however, that other approximations may be equally plausible, e.g., of the type, $\varepsilon(\mu)=\varepsilon_{0}(1-\mu)^{\beta}, \beta>1$.) In such an approximation (e.g., $\beta=1$ ) we obtain a logistic-like equation:

$\frac{\mathrm{d} \mu}{\mathrm{d} \tau}=\varepsilon_{0}\left(\mu-\mu^{2}\right), \quad \mu \geq \mu_{0}$.

This differs from the classic logistic equation commonly used in population ecology (Peters, 1983, p. 124) in that it contains derivative of relative mass $\mu$ over physiological time $\tau \equiv \int_{0}^{\mathrm{t}}\left(B\left(t^{\prime}\right) / E_{\mathrm{m}}\left(t^{\prime}\right) m\left(t^{\prime}\right)\right) \mathrm{d} t^{\prime}$ in Eq. (5) instead of time $t$. Note also that the particular form of Eq. (7) depends on the way one approximates the unknown functional dependence of growth efficiency $\varepsilon$ on relative body mass $\mu$.

Integrating Eq. (7) over $\tau$ we obtain the following expression for the growth curve $\mu(\tau)$ :

$\mu(\tau)=\frac{\mu_{0}}{\mu_{0}+e^{-\varepsilon_{0}\left(\tau-\tau_{0}\right)}\left(1-\mu_{0}\right)}$.
Here $\tau_{0}$ is the physiological age at birth and $\mu_{0} \equiv \mu\left(\tau_{0}\right)$. Growth curves $\mu(\tau)$ will coincide for all organisms sharing similar values of $\varepsilon_{0}$. Inversely, the coincidence of growth curves in different organisms testifies to identical values of $\varepsilon_{0}$.

Using data for 13 species, West et al. (2001) presented a "universal growth curve" predicted by their model. Despite the abundant data on human development, man was not included. Quite expectedly, the model of West et al. (2001) based on relation (1) provides no explanation for the well-known fact that the human growth curve cannot be forced to coincide with growth curves of other organisms by any change of variables (see, for example, Figs. 16.7 and 19.6 in Brody (1945)). This is because Homo sapiens has growth efficiency $\varepsilon_{0}$ from 4 to 7 times lower than other mammals and animals in general, a feature thought to be associated with brain development (Brody, 1945; Gorshkov, 1995, p. 261).

\section{Embryonic development}

The model for embryonic development proposed by Gillooly et al. (2002) is derived from Eq. (1) by neglecting the term $B_{\mathrm{c}} N$. One thus obtains

$\frac{\mathrm{d} m}{\mathrm{~d} t}=\left(\frac{m_{\mathrm{c}}}{E_{\mathrm{c}}}\right) B$,

where $B$ is assumed to scale as $B=B_{0} m^{3 / 4}$ (West et al., 2001; Gillooly et al., 2002). Gillooly et al. claim that the general model (where term $B_{\mathrm{c}} N$ is not ignored in Eq. (1)) is valid for both embryonic and post-embryonic development of organisms. In particular, they assert that constant $a \equiv B_{0} m_{\mathrm{c}} / \mathrm{E}_{\mathrm{c}}$, see Eq. (2), can be determined from the embryonic growth data and used for assessment of post-embryonic growth rates. This essentially means that the value of $B_{0}=$ $a E_{\mathrm{c}} / m_{\mathrm{c}}$ remains constant over the entire life-span of the organism. For the mass-specific metabolic rate $r \equiv B / m$ the model of Gillooly et al. (2002) thus predicts $r / r_{\mathrm{M}}=(m / M)^{-1 / 4}$, where $r_{\mathrm{M}}$ is the mass-specific metabolic rate of an adult organism with mass $M>m$.

We collected data on the mass-specific metabolic rate for four species from four different taxa with mass $M$ ranging over three orders of magnitude (see Fig. 2). During embryonic development, $r$ values do 

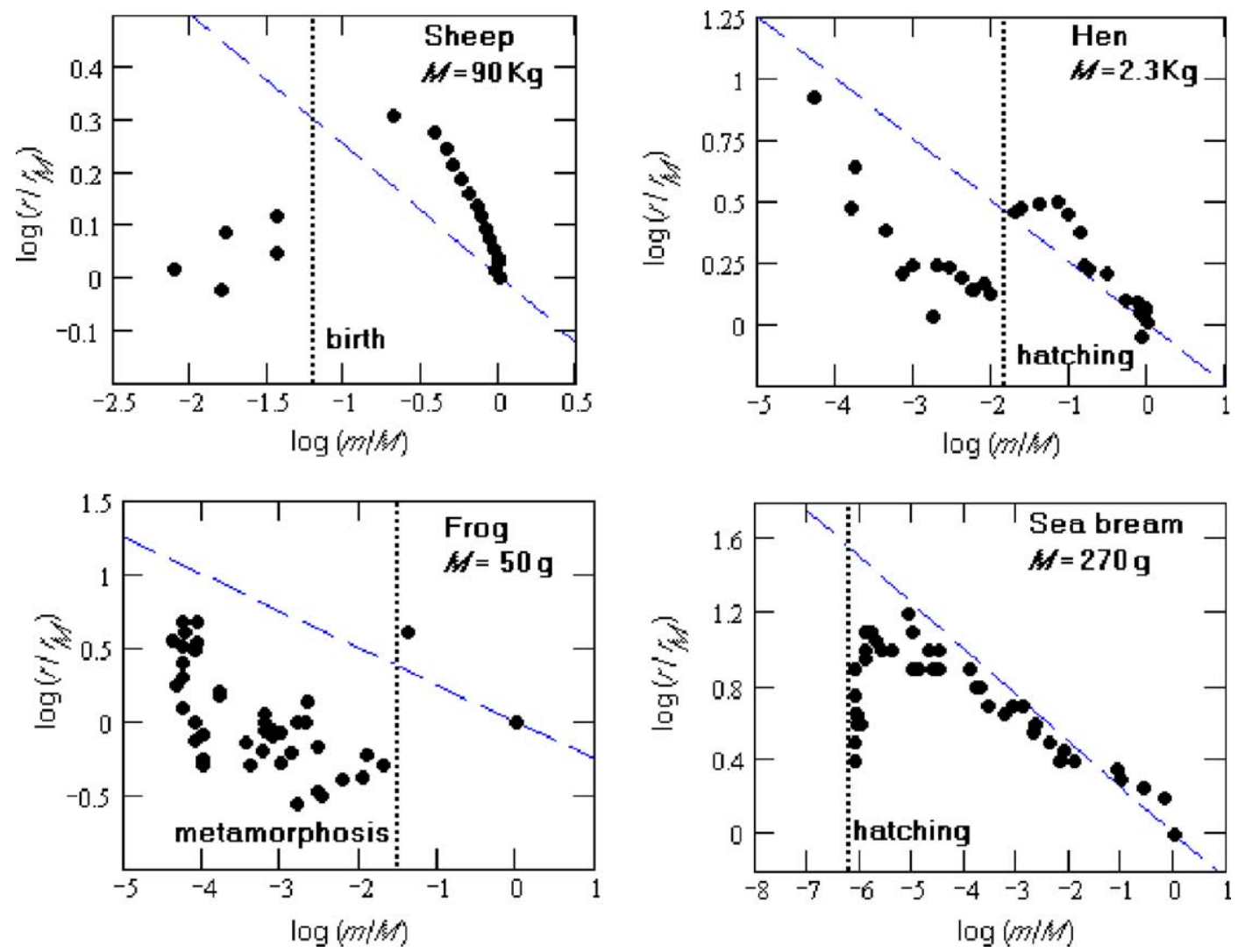

Fig. 2. Relative mass-specific metabolic rates at early to adult developmental stages in various organisms. Here $M$, mass of the largest adult organism shown in the plot; $r_{\mathrm{M}}$, mass-specific metabolic rate of this organism; $r / r_{\mathrm{M}}$, relative mass-specific metabolic rate; $m / M$, relative mass. Dashed lines correspond to dependence $r / r_{\mathrm{M}}=(m / M)^{-1 / 4}$ predicted from the model of Gillooly et al. (2002). Data sources: sheep and hen (Brody, 1945), frog Xenopus laevis (Hastings and Burggren, 1995; Territo and Burggren, 1998), fish Pagrus major (Oikawa et al., 1991). The dotted lines mark transition to juvenile and adult stages. Points to the left of the dotted lines correspond to sheep fetus, chicken embryo, and NF 31-66 stages tadpoles in the frog. In the frog, data for the post-embryonic stage represent averages for 52 and 12 measurements, for froglets and adult frogs, respectively (Hastings and Burggren, 1995). In sea bream, data for early and late larvae, juveniles and adults are presented.

not display a single scaling pattern. During juvenile and adult stages, $r$ displays a more or less monotonous decrease with mass. However, shortly before or after transition to the juvenile stage (e.g., birth in mammals, hatching in fish and birds, metamorphosis in amphibia) all species show a dramatic rise in $r$ values, up to one order of magnitude. Qualitatively, the same abrupt rise of metabolic rate during transition from embryonic to post-embryonic stages was also recorded for sharks (Tullis and Petterson, 2000), crabs (Taylor and Leelapiyanart, 2001) and many altricial birds (Pearson et al., 1999). It is clear from Fig. 2 that the dependence $r / r_{\mathrm{M}}=(m / M)^{-1 / 4}$ predicted by the model of Gillooly et al. (2002) does not fit the experimental data.

Transition from the embryonic to post-embryonic stage is accompanied by sharp changes in the feeding regime. This results in a drop of growth efficiency from $\varepsilon_{\max } \sim 0.7$ (Table 1, Fig. 1) to $\varepsilon_{0} \sim 0.2-0.4$ in the early juvenile post-embryonic stage (Brody, 1945; Pandian and Vernberg, 1987, Fig. 1). As is clear from Fig. 2, in order to sustain normal functioning and growth, the organism chooses to compensate for the drop in growth efficiency by increasing metabolic activity, which is manifested as the observed rise in $r$ values. 
Assuming that organisms aim to sustain their growth rates by increasing metabolic rates, it can be expected that the magnitude of the abrupt drop in proportionality coefficient $\varepsilon /(1-\varepsilon)$ between the growth rate and metabolic rate, Eq. (4), is correlated to the magnitude of the observed rise in $B=\mathrm{rm}$ value, which signifies a relatively small change in body mass. The average embryonic growth efficiency in fish amounts to $\varepsilon_{\max }$ fish $\sim 0.8$ (Table 1) and drops to approximately 0.4 in subsequent developmental stages (Brett and Groves, 1979). This corresponds to a six-fold decrease in $\varepsilon /(1-\varepsilon)$ and compares favorably to the five-fold rise in the $B=r m$ value observed in the fish Pagrus major immediately after hatching, if one assumes that body mass changes only negligibly before and after hatching, Fig. 2. In chicks, growth efficiency drops from 0.67 in a $30-\mathrm{g}$ pre-hatching embryo to 0.26 in a 53-g animal (Brody, 1945, p. 53; Table 1), while the $r$ value rises two-fold, Fig. 2. Thus, $\varepsilon /(1-\varepsilon)$ in chicks decreases 5.8 -fold, to be compensated by a 3.5 -fold rise in $B=\mathrm{rm}$ value. We do not have data on pre-natal growth efficiency of sheep or growth efficiency of frogs in the non-embryonic stages. Although the accuracy of the data analyzed is insufficient to draw unambiguous conclusions, the data point in the direction of dampening of possible abrupt changes in growth rate during important ontogenetic transitions in various organisms. Further studies are definitely needed to explore the degree of generality of this pattern.

Data for growth efficiency displayed in Fig. 1 show a more rapid decrease in post-embryonic $\varepsilon$ values for cows (circles) as compared to hens (squares) at low values of relative mass $\mu$. This change presumably reflects the switch in the energy consumption regime of mammalian juveniles, from lactation to independent feeding. Interestingly, during the early stages of post-natal growth six mammalian species were reported to have metabolic rates increasing appreciably faster than the conventional $B \propto m^{\alpha}$ dependence, where $\alpha \sim 0.6-0.8$ (Poczopko, 1979). For example, for rabbits growing from 70 to $180 \mathrm{~g} \alpha \sim 1.5$ and decreases down to 0.7 for $m>200 \mathrm{~g}$. One may speculate whether this accelerated rise of metabolic rate is to compensate the steep decrease in mammalian growth efficiency observed in early periods of post-natal growth, Fig. 1, or these are two independent processes.
Another instructive example is the comparison of growth efficiency and metabolic rates in organisms with different feeding habits. In biochemical terms it might be cheaper to convert organic matter of one animal to organic matter of another animal than to convert plant organic matter to animal organic matter. Thus, carnivores are expected to display higher growth efficiency than herbivores, as is the case, for example, in fish (Brett and Groves, 1979). Juveniles of grazing mammals should have higher growth efficiency during lactation, when they consume animal proteins (milk), than after weaning, when they start to consume plant organic matter, as exemplified by the data for cows (see Fig. 1). It might be interesting to compare metabolic rates in organisms with different food habits, to find out whether the presumed increase in growth efficiency of organisms feeding on animal biomass is paralleled by decrease in metabolic rates. An analysis of metabolic rates in fructivorous versus insectivorous bats (16 and 15 species, respectively) showed that insectivorous bats do display metabolic rates significantly lower than those of fructivorous bats of similar size (McNab, 1980). Although evidence for other mammalian orders is reported to be less conclusive (McNab, 1986; Elgar and Harvey, 1987) and no effects of diet on metabolic rate were observed in a global study of birds (Bennett and Harvey, 1987), we note that the possible dietary impact on metabolic rate can be overlooked, when metabolic rates of ecologically distant organisms (e.g., rodents and pinnipeds) are compared with an average metabolic dependence for the larger taxon as a whole (e.g., mammals). The dietary impact can be expected to be more pronounced if comparisons are made among organisms of similar ecological design (e.g., within one order, as in $\mathrm{McNab}$ (1980)).

\section{Temperature effects}

For embryonic growth one can approximately put $\varepsilon \approx \varepsilon_{\max }$ and integrate Eq. (4) from $m=0$ at $t=0$ to $m(t)$, assuming traditionally $B=B_{0} m^{\alpha}, \alpha<1$ :

$\frac{t}{m^{1-\alpha}}=\frac{1}{1-\alpha} \frac{1-\varepsilon_{\max }}{\varepsilon_{\max }} \frac{E_{\mathrm{m}}}{B_{0}}$

Metabolic processes accelerate exponentially with temperature according to the well-known Boltz- 
mann distribution, see, e.g., Gorshkov (1995), $\mathrm{B}_{0} \propto$ $\mathrm{e}^{-E_{\mathrm{a}} / k T}$, where $E_{\mathrm{a}}$ is the average activation energy for biochemical reactions of decomposition of organic matter. For temperature changes $T_{\mathrm{c}}$ around a reference temperature $T_{0}$ one obtains $B_{0}\left(T_{\mathrm{c}}\right)=$ $B_{0}\left(T_{0}\right) \exp \left[\left(E_{\mathrm{a}} / k T_{0}^{2}\right)\left(T_{\mathrm{c}} /\left(1+T_{\mathrm{c}} / T_{0}\right)\right)\right]$ (see, e.g., Gillooly et al. (2002)). Gillooly et al. (2002) set $T_{0}=273 \mathrm{~K}$ and $T_{\mathrm{c}} \equiv T-T_{0}$, where $T$ is temperature in Kelvin. Taking the logarithm of both sides of Eq. (10), one obtains a straight line describing dependence between $\left(T_{\mathrm{c}} /\left(1+T_{\mathrm{c}} / T_{0}\right)\right)$ and $\ln \left(t / m^{1-\alpha}\right)$. The slope of this line equals $-E_{\mathrm{a}} / k T_{0}^{2}$ for any value of $\alpha$.

The logarithmic intercept $I$ of this line equals $\ln C$, where

$C \equiv \frac{1}{1-\alpha} \frac{1-\varepsilon_{\max }}{\varepsilon_{\max }} \frac{E_{\mathrm{m}}}{B_{0}\left(T_{0}\right)}$

Taking $\alpha=3 / 4$, Gillooly et al. (2002) provide plots of $\ln \left(t / m^{1 / 4}\right)$ versus $\left(T_{\mathrm{c}} /\left(1+T_{\mathrm{c}} / T_{0}\right)\right.$ for several groups of organisms, where $t$ is the time of embryonic development and $m$ is mass at hatching. They claim that, according to their model, all taxa should display similar logarithmic intercepts $I$, due to the presumed fundamental nature of $B_{0}$ and $E_{\mathrm{c}}$.

For the five plots shown by Gillooly et al. (2002), five values of logarithmic intercept $I$ are obtained, ranging from $I_{\text {fish }}=4.59$ in marine fish to $I_{\text {ins }}=$ 6.55 in multivoltine aquatic insects, $\Delta I=1.96$. These values, with maximum relative difference $\left(I_{\text {ins }}-I_{\text {fish }}\right) / I_{\text {fish }}=0.43$, are interpreted by Gillooly et al. (2002) as sufficiently similar to be supportive of their model. However, as far as $C$ (Eq. (11)) is not dimensionless (unit time per unit mass), the absolute value of the logarithmic intercept $I=\ln C$ changes with the measurement units of $t$ and $m$, having therefore no independent meaning. It is only the difference between any two logarithmic intercepts that is invariant. For example, if Gillooly et al. (2002) increase their units of time by $\mathrm{e}^{4.59} \approx 100$ (e.g., measure time in hundred days instead of days as they do), they obtain values of logarithmic intercepts from $I_{\text {fish }}=0$ to $I_{\text {ins }}=6.55-\Delta I=1.96$. Since the relative difference $\left(I_{\text {ins }}-I_{\text {fish }}\right) / I_{\text {fish }}$ between these values is infinite, they cannot be in any way characterized as similar. It is only the absolute difference of the intercept values for different plots that is invariant with respect to changes of measurement units.
The observed difference between the logarithmic intercepts for the five plots, $\Delta I=I_{\text {ins }}-I_{\text {fish }}=1.96$ contains information about $\mathrm{e}^{1.96} \approx 7$-fold variation in the value of $C$ (Eq. (11)) for embryonic development in the taxa studied. The model of Gillooly et al. (2002) cannot explain this variation. In the meantime, Eqs. (10) and (11) appear to be powerful tools for analyzing these differences. As can be seen from Table 1, development of fish eggs is characterized by the highest $\varepsilon$ values, $\varepsilon_{\max }$ fish $\sim 0.8$ as compared to the average of $\varepsilon_{\max } \sim 0.6$ in the other taxa. From Eq. (11) we predict that, other factors being equal, this should result in a $\left[\left(1-\varepsilon_{\max }\right) / \varepsilon_{\max }\right] /\left[\left(1-\varepsilon_{\max }\right.\right.$ fish $) / \varepsilon_{\max }$ fish $]=$ 2.7-fold decrease in $C_{\text {fish }}$ as compared to other taxa, which is in satisfactory agreement with empirical data discussed by Gillooly et al. (2002). According to these data, the lowest logarithmic intercept is recorded for marine fish eggs in natural environments, for which constant $C_{\text {fish }}=\exp \left(I_{\text {fish }}\right)$ appears to be at least three times lower than for other taxonomic groups (Gillooly et al., 2002).

During post-embryonic development, organisms consume food from the environment which is not designed specifically for their needs, in contrast to egg food stores. Thus, for post-embryonic development $\varepsilon \ll \varepsilon_{\max }$, Fig. 1. For early post-embryonic growth with $\varepsilon \sim 0.2-0.4$ (Brody, 1945; Pandian and Vernberg, 1987) one should obtain values of $C$ about $[(1-\varepsilon) / \varepsilon] /\left[\left(1-\varepsilon_{\max }\right) / \varepsilon_{\max }\right] \approx 3.5-9.4$ times higher as compared to the embryonic growth, which is characterized by an average value of $\varepsilon_{\max } \sim 0.7$ (Table 1 ). This corresponds to $\Delta I=(\ln 3.5)-(\ln 9.4) \approx$ $1.3-2.2$ difference in the values of logarithmic intercept between post-embryonic and embryonic development. In reasonable agreement with this prediction (retrieved from Eq. (10)), the difference in logarithmic intercept values for post-embryonic and embryonic development of zooplankton constitutes $\Delta I=0.8$. The difference between logarithmic intercept for post-embryonic development of zooplankton and the mean value of logarithmic intercept for embryonic development in all the five groups of organisms studied by Gillooly et al. (2002) constitutes $\Delta I=1.4$.

Notably, while devoting much time to explanation of the relatively minor difference $\Delta I=0.8$ between logarithmic intercepts for embryonic and post-embryonic development of zooplankton, Gillooly et al. (2002) say nothing about the more significant 
scatter in logarithmic intercept values, $\Delta I=1.95$, for embryonic development in different taxa, which, as we have shown, can be explained by different values of growth efficiency $\varepsilon_{\max }$ in different taxa.

\section{Conclusions and discussion}

Theoretical studies of ontogenetic growth can be at a large scale characterized as efforts aimed on finding the functional dependence between the rate at which the organism is growing, $\mathrm{d} m / \mathrm{d} t$, and its current value of body mass, $m$. In its general form, such a dependence can be written as

$\frac{\mathrm{d} m}{\mathrm{~d} t}=f\left(m, x_{1}, \ldots x_{n}\right)$,

where $m$ is body mass, while $x_{1}, \ldots, x_{n}$ are a number of $m$-independent parameters. The expectation that such dependence exists is based on the observations that the major energetic characteristics of the organism like food consumption and metabolic rate in many cases appear to be (allometric) functions of body mass.

One can distinguish between two principally different approaches to the task of finding $f\left(m, x_{1}, \ldots, x_{n}\right)$. For definiteness sake, everywhere below we refer to them as formal modeling and theoretical modeling, although more comprehensive terms can be presumably thought of. In formal modeling studies, one looks for a mathematical function that could provide a good fit for the observed growth curves of a particular organism or a group of organisms. From the mathematical point of view, it is obvious that a variety of formal functions can be found that could fit the observed growth curves in different organisms. For example, Peters (1983, p. 123) lists three different functions $f\left(m, x_{1}, \ldots, x_{n}\right)$, including that of von Bertalanffy (Eq. (2)), noting that they are all equally good descriptors of the data. An essential feature of formal modeling approach is that at least some of parameters $x_{1}, \ldots, x_{n}$ can be only obtained by fitting the selected function to the available data rather than by direct measurements. These parameters are formal in that sense that they do not correspond to any independently measured physical or biological constants.

For example, the formal model of West et al. (2001), where $f(m, a, b)=a m^{3 / 4}-b m$, see Eq. (2), provided a reasonably good description of the growth curves the authors presented, after parameters $a$ and $b$ have been fitted to the data. However, the attempt of West et al. (2001) to interpret these parameters as functions of measurable quantities (e.g., energy content of the cell) immediately resulted in violation of the energy conservation law (see Section 1) and heat imbalance for homoiothermic animals (see Section 2).

After all parameters of the model are fitted, a formal model may yield very precise descriptions of those particular data it was designed to fit. However, within the formal modeling approach one has no grounds to expect the same mathematical function to describe growth curves of different organisms. As a rule, generality in the formal modeling approach is therefore achieved by increasing the number $n$ of formal parameters used in the model. For example, as we have shown in Section 2, constant coefficient $b$ in Eq. (2) leads to energetic controversy when Eq. (2) is applied to homoiothermic animals. In order to retain the model structure of the type $\mathrm{d} m / \mathrm{d} t=c_{1} m^{\alpha}-c_{2} m^{\beta}(0<\alpha<$ $\beta$ ), for homoiothermic animals one would have therefore to introduce additional formal parameters specifically accounting for homoiothermy (see, e.g., heating length parameter in Kooijman (2000)). Fitting parameters of one formal model makes no sense within the framework of the other; as such parameters are retrieved from fitting the data by different mathematical functions.

As can be expected, formal fitting functions are deprived of independent predictive power. In this paper we have demonstrated the inadequacy of Eqs. (1) and (2) for explaining (a) the organismal energy budget during embryonic development (Section 1); (b) the human growth curve (Section 3); (c) patterns of metabolic rate change during transition from embryonic to post-embryonic stages (Section 4); and (d) differences between parameters describing embryonic development in different taxa (Section 5).

The major goal of what may be called theoretical modeling consists in revealing correlations between variables that can be experimentally measured. Such variables constitute the domain of objective scientific information about the world and conform to the fundamental equations like those of energy and matter conservation (Brillouin, 1956). In this paper we used ontogenetic growth equations, see Eqs. (4) and (5), derived from the conservation of energy Eq. (3) for the growing organism, formulated in terms of three mea- 
surable ecological variables: body mass $\mathrm{m}$ (or cumulative energy content $E$ ), metabolic rate $B$, and growth efficiency $\varepsilon$. We have shown how Eqs. (4) and (5) explain these data whereas the formal models of West et al. (2001) and Gillooly et al. (2002) failed.

The idea that a successful theory cannot not include immeasurable (unobservable) quantities was responsible for the explosion-like development of physics in the 20th century. Heisenberg (1925) wrote that "relationships between quantities that are apparently unobservable in principle, e.g., position and period of revolution of the electron", cannot constitute the basis of a successful theory. As we now know, the exclusion of such unobservable quantities from quantum physics theory led to emergence of a conceptually new scientific vision of the world. Within the theoretical modeling approach, correlations found between measurable variables have absolute significance and are independent of mathematical formalism that is used for their description (Brillouin, 1956). This advantage of the theoretical approach over formal modeling was appreciated by West et al. (2001), who wrote about the need for elaborating models justified on fundamental biological mechanisms rather than merely on goodness of fit.

However, the models of West et al. (2001) and Gillooly et al. (2002) did not develop into a theoretical framework, as they were formulated in terms of immeasurable quantities and, consequently, based on non-testable assumptions.

This can be illustrated by the assumption of constant maintenance expenses $B_{\mathrm{c}}$, see Eq. (1), which is adopted in a number of other ontogenetic growth models as well. The measurable quantity describing an animal's metabolism is its metabolic rate $B$. It can be formally thought of as consisting of different parts, like maintenance expenses, growth expenses, metabolic work, etc. (see, e.g., Kooijman, 2000). However, empirical separation of these parts represents a well-known and practically irresolvable problem (Brody, 1945). For example, the maintenance expenses in animals are commonly measured in laboratory conditions under various degrees of starvation, i.e., by attenuating the food supply until growth is depressed and all energy consumed by the animal can be thought of as maintaining the existing biomass (Hawkins et al., 1989; Nisbet et al., 2000). However, "one may question the significance of maintenance as obtained for a non-growing organism in relation to the maintenance cost of a normally growing one" (Brody, 1945, p. 43). The very setting up of experiments of such kind assumes that the organism can be viewed as switching freely between growing and non-growing regimes, so that its metabolic rate is a simple additive quantity composed of maintenance and growth expenses. In reality, if one artificially suppresses growth of a juvenile organism (e.g., by starvation), this will most likely result in significant distortions of its development or even death. This means that for a developing juvenile organism normal "maintenance" is unthinkable without growth, so that any objective division of metabolic rate into growth and maintenance expenses is impossible. On the other hand, increase of biomass of an adult organism (e.g., due to overfeeding) has little to do with genetically programmed ontogenetic growth and may have quite different metabolic manifestations as compared to the former. Similarly, it is entirely subjective whether to attribute metabolic work (e.g., walking, food gathering, etc.) to maintenance expenses or not. For example, even if maintenance expenses are interpreted in a narrow sense as biochemical maintenance of living cells, one may note that walking and other physical exercises are necessary for normal oxygen supply of cells. If physical activities of the organism are artificially suppressed for an extended period, this results in pathologic changes of its health condition, which could propagate down to the cellular level.

We sum up that maintenance expenses remain an immeasurable quantity. It is legitimate therefore that the model of Gillooly et al. (2002) based on the non-testable assumption of constant mass-specific maintenance expenses was unable to predict the abrupt rise of the measurable mass-specific metabolic rate $r \equiv B / m$ observed in different organisms during transition from embryonic to post-embryonic developmental stages (see Fig. 2). As discussed above (Section 4), this change is at least partially compensated by the drop of growth efficiency $\varepsilon$, so that the growth rate $\mathrm{d} m / \mathrm{d} t$ (see Eq. (4)) does not change significantly during such transitions. Thus, if one formally assumes $\mathrm{d} m / \mathrm{d} t=a m^{3 / 4}$ (see Eq. (9)), where $a$ is a constant (Gillooly et al., 2002) and monitors $m$ and $\mathrm{d} m / \mathrm{d} t$ only, one completely overlooks the fundamental changes in metabolic rate $B$ and growth 
efficiency $\varepsilon$ that accompany transitions to juvenile stages, although the formal fit of the growth curves by Eq. (9) may remain very good. If, on the contrary, one operates with measurable variables, for example, with $\mathrm{d} m / \mathrm{d} t$ and $B$, one can predict the drop of ecological efficiency $\varepsilon$. Conversely, one can also estimate the rise in mass-specific metabolic rate from the known magnitude of $\varepsilon$ change.

One finding that we consider remarkable is that in different animals the growth efficiency $\varepsilon$ seems to display a universal dependence on relative body mass $\mu=m / M$ (Brody, 1945; Pandian and Vernberg, 1987, Fig. 1), although very few detailed studies are currently available. Using the scarce data available, we showed that the dependence $\varepsilon(\mu)$ can be reasonably approximated by a linear function $\varepsilon(\mu)=\varepsilon_{0}(1-\mu)$, where $\varepsilon_{0} \sim 0.2$ is the value of growth efficiency at birth. Another magnitude that suggests itself for the role of a fundamental parameter in ontogenetic modeling is the value of growth efficiency during embryonic growth, which on average amounts to $\varepsilon_{\max } \sim$ 0.7 (see Table 1). Values of $\varepsilon_{0}$ and $\varepsilon_{\max }$ are fundamental in that they characterize the efficiency of energy conversion within the living world, which is determined by the way living matter is organized and cannot be predicted a priori. More studies are needed to investigate the degree of universality of these values.

In conclusion, we believe that further empirical and theoretical investigations of the functional dependence of growth efficiency on relative body mass, as well as of a possible negative correlation between growth efficiency and metabolic rate (Section 4), may lead to important advancements in the theory of ontogenetic growth and make it ultimately possible to formulate function $f\left(m, x_{1}, \ldots, x_{n}\right)$ (Eq. (12)) using ecological parameters that are physically and biologically sound and independently measurable.

\section{Acknowledgements}

Peter Morrell kindly provided editorial comments and suggestions for this manuscript. This work is supported by the Russian Foundation for Basic Research and U.S. National Science Foundation and the University of California Agricultural Experiment Station.

\section{References}

Arnould, J.P.Y., Boyd, I.L., Socha, D.G., 1996. Milk consumption and growth efficiency in Antarctic fur seal Arctocephalus gazella pups. Can. J. Zool. 74, 254-266.

Bennett, P., Harvey, P., 1987. Active and resting metabolism in birds-allometry, phylogeny and ecology. J. Zool. 213, 327363.

Brett, J.R., Groves, T.D.D., 1979. Physiological energetics. In: Hoar, W.S., Randall, D.J., Brett, J.R. (Eds.), Fish Physiology, Bioenergetics and Growth, vol. 8. Academic Press, New York, pp. 279-352.

Brillouin, L., 1956. Science and Information Theory. Academic Press, New York.

Brody, S., 1945. Bioenergetics and Growth. Reinhold Publishing Corporation, New York.

del Giorgio, P.A., Cole, J.J., 1998. Bacterial growth efficiency in natural aquatic systems. Ann. Rev. Ecol. Syst. 29, 503-541.

Economos, A.C., 1982. On the origin of biological similarity. J. Theor. Biol. 94, 25-60.

Elgar, M.A., Harvey, P.H., 1987. Basal metabolic rates in mammals: allometry, phylogeny and ecology. J. Funct. Ecol. 1, $25-36$.

Gillooly, J.F., Charnov, E.L., West, G.B., Savage, V.M., Brown, J.H., 2002. Effects of size and temperature on developmental time. Nature 417, 70-73.

Gorshkov, V.G., 1995. Physical and Biological Bases of Life Stability. Man, Biota, Environment. Springer, Berlin.

Hastings, D., Burggren, W., 1995. Developmental changes in oxygen consumption regulation in larvae of the South African clawed frog Xenopus laevis. J. Exp. Biol. 198, 2465-2475.

Hawkins, A.J.S., Widdows, J., Bayne, B.L., 1989. The relevance of whole-body protein metabolism to measured costs of maintenance and growth in Mytilus edulis. Physiol. Zool. 62, 745-763.

Heisenberg, W., 1925. Ueber quantentheoretische Umdeutung kinematischer und mechanischer Beziehung. Zeitschr. f. Phys. 33, 879-883.

Kooijman, S.A.L.M., 2000. Dynamic Energy and Mass Budgets in Biological Systems. Cambridge University Press, Cambridge.

McNab, B.K., 1980. Food habits, energetics, and the population biology of mammals. Am. Nat. 116, 106-124.

McNab, B.K., 1986. The influence of food habits on the energetics of eutherian mammals. Ecol. Monogr. 56, 1-19.

Nisbet, R.M., Muller, E.B., Lika, K., Kooijman, S.A.L.M., 2000. From molecules to ecosystems through dynamic energy budget models. J. Anim. Ecol. 69, 913-926.

Odum, E.P., 1971. Fundamentals of Ecology, 3rd ed. W.B. Sounders, Philadelphia.

Oikawa, S., Itazawa, Y., Gotoh, M., 1991. Ontogenetic change in the relationship between metabolic rate and body mass in a sea bream Pagrus major (Temmink \& Schlegel). J. Fish Biol. 38, 483-496.

Pandian, T.J., Vernberg, F.J. (Eds.), 1987. Animal Energetics. Academic Press, San Diego.

Parra, G., Yúfera, M., 2001. Comparative energetics during early development of two marine fish species, Solea senegalensis (Kaup) and Sparus aurata (L.). J. Exp. Biol. 204, 2175-2183. 
Pearson, J.T., Noma, Y., Tazawa, H., 1999. Developmental patterns of heart rate in altricial avian embryos and hatchlings. J. Exp. Biol. 202, 1545-1550.

Peters, R.H., 1983. The Ecological Implications of Body Size. Cambridge University Press, Cambridge.

Pike, R.L., Brown, M.L., 1975. Nutrition: An Integrated Approach. Wiley, New York.

Poczopko, P., 1979. Metabolic rate and body size relationships in adult and growing homeotherms. Acta Theriol. 24, 125-136.

Taylor, H.H., Leelapiyanart, N., 2001. Oxygen uptake by embryos and ovigerous females of two intertidal crabs, Heterozius rotundifrons (Belliidae) and Cyclograpsus lavauxi (Grapsidae): scaling and the metabolic costs of reproduction. J. Exp. Biol. 204, 1083-1097.

Territo, P.R., Burggren, W.W., 1998. Cardio-respiratory ontogeny during chronic carbon monoxide exposure in the clawed frog Xenopus laevis. J. Exp. Biol. 201, 1461-1472.
Tullis, A., Petterson, G., 2000. Growth and metabolism in the embryonic white-spotted bamboo shark Chiloscillum plagiosum: comparison with embryonic birds and reptiles. Physiol. Biochem. Zool. 73, 271-282.

von Bertalanffy, L., 1957. Quantitative laws in metabolism and growth. Q. Rev. Biol. 32, 217-231.

West, G.B., Brown, J.H., Enquist, B.J., 2001. A general model for ontogenetic growth. Nature 413, 628-631.

Whittaker, R.H., 1975. Communities and Ecosystems. MacMillan, New York.

Worthy, G.A.J., Lavigne, D.M., 1983. Changes in energy stores during postnatal development of the harp seal, Phoca groenlandica. J. Mammal. 64, 89-96.

Yúfera, M., Parra, G., Santiago, R., Carrascosa, M., 1999. Growth, carbon, nitrogen and caloric content of Solea senegalensis (Pisces: Soleidae) from egg fertilization to metamorphosis. Mar. Biol. 134, 43-49. 